\title{
Chemical imaging of lipid droplets in muscle tissues using hyperspectral coherent Raman microscopy
}

Citation for published version (APA):

Billecke, N., Rago, G., Bosma, M., Eijkel, G., Gemmink, A., Leproux, P., Huss, G., Schrauwen, P., Hesselink, M. K., Bonn, M., \& Parekh, S. H. (2014). Chemical imaging of lipid droplets in muscle tissues using hyperspectral coherent Raman microscopy. Histochemistry and Cell Biology, 141(3), 263-273. https://doi.org/10.1007/s00418-013-1161-2

Document status and date:

Published: 01/01/2014

DOI:

10.1007/s00418-013-1161-2

Document Version:

Publisher's PDF, also known as Version of record

Document license:

Taverne

Please check the document version of this publication:

- A submitted manuscript is the version of the article upon submission and before peer-review. There can be important differences between the submitted version and the official published version of record.

People interested in the research are advised to contact the author for the final version of the publication, or visit the DOI to the publisher's website.

- The final author version and the galley proof are versions of the publication after peer review.

- The final published version features the final layout of the paper including the volume, issue and page numbers.

Link to publication

\footnotetext{
General rights rights.

- You may freely distribute the URL identifying the publication in the public portal. please follow below link for the End User Agreement:

www.umlib.nl/taverne-license

Take down policy

If you believe that this document breaches copyright please contact us at:

repository@maastrichtuniversity.nl

providing details and we will investigate your claim.
}

Copyright and moral rights for the publications made accessible in the public portal are retained by the authors and/or other copyright owners and it is a condition of accessing publications that users recognise and abide by the legal requirements associated with these

- Users may download and print one copy of any publication from the public portal for the purpose of private study or research.

- You may not further distribute the material or use it for any profit-making activity or commercial gain

If the publication is distributed under the terms of Article $25 \mathrm{fa}$ of the Dutch Copyright Act, indicated by the "Taverne" license above, 


\title{
Chemical imaging of lipid droplets in muscle tissues using hyperspectral coherent Raman microscopy
}

\author{
Nils Billecke • Gianluca Rago • Madeleen Bosma • Gert Eijkel • Anne Gemmink • \\ Philippe Leproux • Guillaume Huss • Patrick Schrauwen • Matthijs K. C. Hesselink • \\ Mischa Bonn · Sapun H. Parekh
}

Accepted: 22 October 2013 / Published online: 2 November 2013

(C) Springer-Verlag Berlin Heidelberg 2013

\begin{abstract}
The accumulation of lipids in non-adipose tissues is attracting increasing attention due to its correlation with obesity. In muscle tissue, ectopic deposition of specific lipids is further correlated with pathogenic development of insulin resistance and type 2 diabetes. Most intramyocellular lipids are organized into lipid droplets (LDs), which are metabolically active organelles. In order to better understand the putative role of LDs in pathogenesis, insight into both the location of LDs and nearby chemistry of muscle tissue is very useful. Here, we demonstrate the use of label-free coherent anti-Stokes Raman scattering (CARS) microscopy in combination with multivariate, chemometric analysis to visualize intracellular lipid accumulations in
\end{abstract}

Nils Billecke and Gianluca Rago have contributed equally to this work.

Electronic supplementary material The online version of this article (doi:10.1007/s00418-013-1161-2) contains supplementary material, which is available to authorized users.

N. Billecke $\cdot$ G. Rago $\cdot$ M. Bonn $\cdot$ S. H. Parekh $(\bowtie)$

Molecular Spectroscopy Department, Max Plank Institute

for Polymer Research, Ackermannweg 10, 55128 Mainz,

Germany

e-mail: parekh@mpip-mainz.mpg.de

\section{G. Rago · G. Eijkel}

FOM Institute AMOLF, Science Park 104, 1098 XG Amsterdam, The Netherlands

G. Rago

Deloitte Consulting B.V., Laan van Kronenburg 2,

1183 AS Amstelveen, The Netherlands

M. Bosma $\cdot$ P. Schrauwen

Department of Human Biology, School for Nutrition, Toxicology and Metabolism, Maastricht University Medical Center,

6200 MD Maastricht, The Netherlands ex vivo muscle tissue. Consistent with our previous results, hyperspectral CARS microscopy showed an increase in LDs in tissues where LD proteins were overexpressed, and further chemometric analysis showed additional features morphologically (and chemically) similar to mitochondria that colocalized with LDs. CARS imaging is shown to be a very useful method for label-free stratification of ectopic fat deposition and cellular organelles in fresh tissue sections with virtually no sample preparation.

Keywords Lipid droplet $\cdot$ Microscopy $\cdot$ Chemical imaging $\cdot$ Raman spectroscopy $\cdot$ Multivariate analysis · Hyperspectral

\section{Introduction}

Obesity is one of the major health risks in Western countries, with an increasing prevalence above 1.4 billion adults

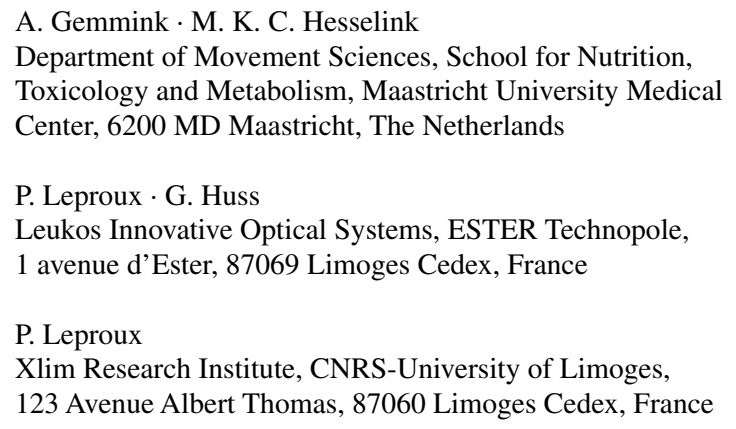


worldwide (World Health Organisation). One associated pathologic outcome of chronic obesity is insulin resistance, which is a first step in the development of type 2 diabetes. While the exact cause of insulin resistance is unclear, fat accumulation in non-adipose tissues (ectopic fat storage) is likely to play a role (Lee et al. 2009). Ectopically stored lipids in tissues such as liver and skeletal muscle are predominantly packed in discrete lipid droplets (LDs). Skeletal muscle LDs contain so-called intramyocellular lipids (IMCL), and elevated IMCL has been correlated with reduced insulin sensitivity in previous in vivo and in vitro investigations (Aguer et al. 2010; Goodpaster et al. 2000). This correlation potentially links ectopic fat deposition in skeletal muscle with the onset of insulin resistance and the transition to type 2 diabetes.

Intramyocellular lipids in LDs comprise esterified fats, cholesterol, and other lipid species, many of which are derivatives of fatty acids (FAs) with varying chain length and level of saturation. The specific lipid composition of intramuscular LDs has been shown to profoundly affect their pathological potential. While elevated levels of intramuscular triacylglycerides (TAGs) do not necessarily lead to insulin resistance (Goodpaster et al. 2001), accumulation of specific lipids such as sphingolipids, especially ceramides, has repeatedly been linked to insulin resistance in muscle (Chavez et al. 2003; Stratford et al. 2004). Moreover, it has been speculated that the subcellular spatial distribution and interorganelle interaction of LDs play a role in the insulin-desensitizing effect (Nielsen et al. 2010; Shaw et al. 2008; Sollner 2007). To better understand the link between ectopic lipid accumulation and insulin desensitization, detailed information on the composition, biochemistry, and cellular localization of LDs is highly desirable.

Classically, evaluation of lipid species in tissues involves extraction and subsequent gas chromatography to quantitatively determine the amount of each individual chemical compound present in a sample (Seppanen-Laakso et al. 2002); regrettably, loss of all spatial information is inevitable in this type of analysis. More recently, mass spectroscopic imaging methods such as matrix-assisted laser desorption ionization-imaging mass spectrometry (MALDI-IMS) and time-of-flight secondary ion mass spectrometry (ToF-SIMS) have emerged as attractive compromises between spatial localization and chemical specificity. MALDI-IMS permits detection with high sensitivities (femto- to attomolar) in a local region of the sample ( 3-10 $\mu \mathrm{m}$ voxel size) for a large range of masses (from $\sim 100 \mathrm{Da}$ to $\sim 300 \mathrm{kDa}$ ). However, achieving such high resolution requires careful matrix embedding and sample preparation, which may affect tissue structure and localization of biomolecules. Furthermore, the spatial resolution is insufficient to interrogate individual myocellular LDs (0.05-3 $\mu \mathrm{m}$ diameter) at this time (Rompp and Spengler 2013). On the contrary, ToF-SIMS that uses a high-power ion beam is focused in the sample causing ejection of atoms and molecular fragment as well as secondary ions, the latter being then resolved in a ToF analyzer. Given that a focused ion beam is used for ionization, the spatial resolution on the 100-nm scale is theoretically available in SIMS; however, practical limitations have limited the spatial resolution to $\sim 1 \mu \mathrm{m}$ for biological samples. Similar to MALDI-IMS, ToF-SIMS has enormous power for molecular identification of relatively low molecular weight species (up $10 \mathrm{kDa}$ ) but is destructive and requires sample preparation that is not trivial for maintaining the sample integrity (Boxer et al. 2009; Fletcher and Vickerman 2013). The probing depth for secondary ion detection is $1-2 \mathrm{~nm}$ below the surface, which makes applications to standard (clinical) histological samples more challenging due to the relatively thick (micron) slices.

Studies focused on LD localization, tissue composition, and interorganelle interaction within fresh tissues are scarce as suitable techniques capable of simultaneously addressing these parameters are not prevalent. While immunohistochemistry (IHC) approaches provide useful information on interorganelle interaction between LDs, LD spatial distribution, and LD size, this requires specific labeling of the distinct components. Fluorescent IHC typically supports up to three or four probes to be imaged simultaneously, ultimately limited by the probe emission spectra and microscopy instrumentation. A few approaches exist for localization of bulk protein and lipid staining for tissue stratification. For lipid imaging, fluorescent and chromophore probes can provide localization information about the presence or absence of lipids in the sample; however, visualization of all lipid species in a sample is heavily dependent on sample preparation. Unfortunately, similar holistic imaging of all proteins in either tissue or cell samples is quite challenging with IHC approaches. While IHC provides very useful information for specific macromolecular and organelle distribution, localization of whole molecular classes (e.g., all proteins, DNA, and lipids) and their organization relative organelles is not trivial.

Thus, to further elucidate the role of intramyocellular LDs in the development of insulin resistance and type 2 diabetes, a method is required that combines quantitative spatial information about LD size and content at submicron resolution, local chemical composition of the sample, and the ability to examine interorganelle interaction. Such requirements are potentially fulfilled by microscopy techniques based on vibrational (chemical) contrast. These approaches use the intrinsic vibrations of molecules for contrast and allow generation of chemically specific images with diffraction-limited optical resolution (Puppels et al. 1990). Spontaneous Raman microscopy is a well-established vibrational (hyperspectral) microscopy techniquesimilar to imaging mass spectrometry-and has been 
(A)

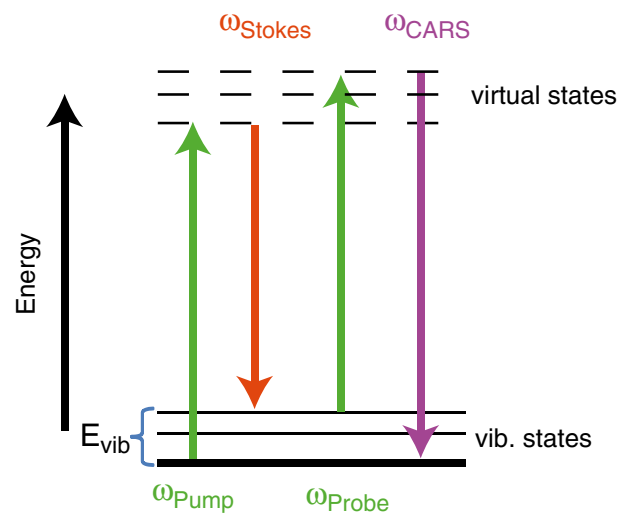

(B)

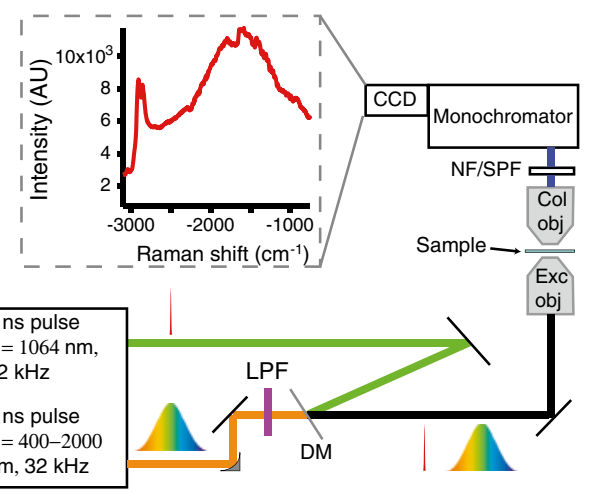

Fig. 1 a Energy diagram of coherent anti-Stokes Raman scattering (CARS). Three photons ( $\omega_{\text {pump }}, \omega_{\text {Stokes }}$, and $\left.\omega_{\text {probe. }}\right)$ interact with the sample to produce a fourth CARS photon $\left(\omega_{\mathrm{CARS}}\right)$. When $\omega_{\text {pump }}-\omega_{\text {Stokes }}=\mathrm{E}_{\mathrm{vib}}$, the process is resonantly enhanced, thereby giving a strong signal corresponding to vibrational modes in the sample, similar to Raman scattering. b Simplified optical schematic of the hyperspectral CARS microscope. The pump and probe photons are from the same laser, $\lambda=1,064 \mathrm{~nm}$, and the Stokes laser spans from

extensively used to study the local chemical composition of LDs in cultured cells (Matthaus et al. 2012; Vaandrager et al. 2009). However, the application of hyperspectral Raman microscopy to study fresh tissue sections has been limited by two principle challenges: (1) the large autofluorescence response of tissues (Wang et al. 2012) and (2) the limited penetration depth of the visible excitation wavelengths (Centonze and White 1998).

Coherent anti-Stokes Raman Scattering (CARS) microscopy overcomes these limitations and has been shown to produce chemically specific images of LD composition in cultured cells (Day et al. 2010). CARS is a multiphoton analog of spontaneous Raman scattering in which anti-Stokes (blue-shifted) light is generated by interaction between a pump, Stokes, and probe photon within the sample (Fig. 1a). If the energy difference between the pump and Stokes photons is resonant with a vibrational mode in the sample, the anti-Stokes light is resonantly enhanced and can be 6 orders of magnitude stronger than spontaneous Raman scattering (Tolles et al. 1977). Because the detected light is blue-shifted, contamination from autofluorescence is minimized in CARS. Furthermore, most implementations use near-IR excitation, which can penetrate several $100 \mathrm{~s}$ of microns due to minimal absorption and scattering relative to visible wavelengths often used in Raman microscopy (Helmchen and Denk 2005; Centonze and White 1998; Weigert et al. 2010).

While hyperspectral CARS microscopy potentially alleviates many data acquisition challenges incurred with

$\lambda=1,100-1,600 \mathrm{~nm}$ after being spectrally filtered (LPF). These beams are combined by a dichroic mirror (DM) and then focused by the excitation objective (Exc obj, 100X, 0.75 NA) into the sample. The CARS light is collected by the collection objective (Col obj, 10X, 0.25 NA), filtered (NF/SPF) from the pump and Stokes lasers, and detected by the monochromator and CCD. The sample is then moved in the $x, y, z$, or directions to the next position, and another spectrum is collected. The insets show a raw CARS spectrum

hyperspectral Raman microscopy, the beneficial qualities of CARS come with data interpretation ambiguity. Until recently, it was impossible to use CARS data quantitatively. We and others have developed mathematical algorithms (Liu et al. 2009; Vartiainen et al. 2006) to render CARS spectra similar to spontaneous Raman spectra, whose signals scale linearly with signal intensity. With quantitative CARS spectra, it is possible to construct images from individual vibrational modes, analogous to imaging mass spectrometry. In addition, multivariate (chemometric) analytical methods, which are well developed for hyperspectral Raman (van Manen et al. 2005; Matthaus et al. 2007) and mass spectrometry imaging, are also applicable to quantitative hyperspectral CARS data (Pohling et al. 2011a, b).

In the present study, we take advantage of the application of hyperspectral CARS microscopy and chemometric analysis to image the chemical composition of myocellular fibers and localize intracellular LDs in excised muscle tissue. We study LDs and composition in tissue sections from a model system where myocellular LD formation was promoted by a nutritional and genetic intervention (via perilipin 5, PLIN5, overexpression) (Bosma et al. 2012). We find that hyperspectral CARS microscopy data from biochemically complex tissue sections can be reliably analyzed with both direct integration of vibrational peaks and unsupervised chemometric analysis. Both analyses produced similar results for LD localization showing greater abundance of LDs in PLIN5-overexpressing fibers. These findings match with data obtained in the same muscles via classical 
morphometry using transmission electron microscopy and immunofluorescence (Bosma et al. 2012). Furthermore, we show that chemometric analysis of the hyperspectral CARS data revealed additional mitochondrial-like features in overexpressing fibers that were invisible with direct peak integration. Together, these data show the combined spatial and chemical specificity of hyperspectral CARS microscopy for label-free, biochemical imaging of tissue samples.

\section{Materials and methods}

\section{Experimental model system}

In the present study, we took advantage of a rat model of augmented LD content. In this particular model, unilateral gene electroporation of a gene involved in LD maintenance (PLIN5) was applied. Briefly, 8-week-old male Wistar rats were fed a high-fat diet (45\% energy from fat, D01060502, Research Diets) for the duration of the 3-week intervention. Two weeks after the start of the diet, overexpression of PLIN5 in either the right or left tibialis anterior (TA) muscle of the rat was accomplished by an in vivo DNA electrotransfer technique to obtain overexpression of mouse PLIN5 in one leg (Bosma et al. 2012); the unsuccessfully transfected fibers in the same TA muscle slice served as an internal control in this study. Rats were killed 8 days postelectroporation. TA muscles were excised and rapidly frozen in melting isopentane. The Animal Care and Use Committee of Maastricht University approved the experiments (approval number 2010-036), and the study complied with the principles of laboratory animal care.

\section{Tissue excision and immunofluorescence}

Frozen TA muscles were transferred to a cryostat and cut transversally into serial sections (5 and $20 \mu \mathrm{m}$ ). After mounting on uncoated glass slides, sections were allowed to dry for $30 \mathrm{~min}$ at room temperature and then stored at $-20{ }^{\circ} \mathrm{C}$ until imaging. Consecutive sections were cut from each sample of muscle tissue. A $20-\mu \mathrm{m}$ section and adjacent $5-\mu \mathrm{m}$ section were used for CARS measurements. A second 5- $\mu \mathrm{m}$ section was used for immunofluorescence. The sections used for CARS were imaged in air directly after warming to room temperature without further processing.

The 5- $\mu \mathrm{m}$ section used for immunofluorescence was stained for lipid droplets, nuclei, and sarcolemmal cell borders. All staining procedures were carried out at room temperature. Sections were fixed for $1 \mathrm{~h}(4 \%$ PFA in PBS, $\mathrm{pH}$ 7.4) and washed three times for $5 \mathrm{~min}$ in PBS. The sections were then incubated with primary antibody against Laminin (L9393, Sigma-Aldrich) diluted 1:80 in AbDil buffer ( $150 \mathrm{mM} \mathrm{NaCl}, 20 \mathrm{mM}$ Tris, and $2 \%$ BSA, pH 7.4) for $1 \mathrm{~h}$ in a humidified chamber. After washing three times with PBS, sections were incubated with an Alexa Fluor 594 secondary antibody (A-11037, Invitrogen) diluted 1:200, $1 \mu \mathrm{g} / \mathrm{mL}$ Bodipy 493/503 (D-3922, Invitrogen), and DAPI diluted 1:1,000 in AbDil buffer. Following three washing steps with PBS, sections were mounted in fluorescence mounting medium (Dako, Glostrup) and imaged on an Olympus IX80 inverted microscope (Olympus) using Cell $\mathrm{F}$ imaging software. Images were processed and overlays created using ImageJ.

\section{Hyperspectral CARS microscopy}

A dual-output laser source (Leukos-CARS, Leukos) provides the pump and Stokes beams. The source is a passively Q-switched 1,064-nm microchip laser, delivering sub-nanosecond pulses at $32 \mathrm{kHz}$ repetition rate and $\sim 300 \mathrm{~mW}$ average power. This beam is divided into two parts internally: one part is introduced into a photonic crystal fiber that creates supercontinuum emission from $420-2,400 \mathrm{~nm}$ for Stokes beam, with more than $100 \mu \mathrm{W} \mathrm{nm}{ }^{-1}$ spectral power density from 1,050 to $1,600 \mathrm{~nm}$, and the second part is used as the pump and probe beam at 1,064 $\mathrm{nm}$. Both beams are provided from the Leukos-CARS source with the Stokes beam emerging from a fiber and the pump beam provided in free space. The Stokes beam is long-pass-filtered to have a bandwidth from 1,100 to $1,600 \mathrm{~nm}$ and routed on the optical table to match pump beam in time and space at the focus of the microscope (Fig. 1b). Each beam has an average power of $\sim 30 \mathrm{~mW}$ at the sample.

The sample was raster-scanned across the focal volume with steps of $0.25 \mu \mathrm{m}$ in plane (Mad City Labs). Large images were reconstructed from adjacent tiles with in-plane dimensions of $20 \times 20 \mu \mathrm{m}$ (corresponding to $81 \times 81$ pixels). For each position in the sample, a CARS spectrum in the range between $-3,100$ and $-600 \mathrm{~cm}^{-1}$ was acquired on a cooled CCD (Andor). CARS spectra were acquired with pixel dwell times of $1 \mathrm{~s}$ (Fig. 1b, inset). The spatial resolution of the instrument was independently measured to be $\sim 0.6 \times 0.6 \times 3.5 \mu \mathrm{m}^{3}$, and the spectral pitch was $\sim 4 \mathrm{~cm}^{-1}$ per CCD pixel.

\section{Raw CARS data analysis}

Raw CARS spectra were analyzed with custom routines in Igor Pro 6.22A (Wavemetrics). Pretreatment of the data with the maximum entropy method (MEM) algorithm retrieves the imaginary component of the third-order Raman susceptibility (Vartiainen et al. 2006), here referred to as Raman-retrieved CARS (RR CARS) spectra. Images of RR CARS modes were generated in Igor Pro. All other image handling was done using ImageJ software. 


\section{Chemometric analysis}

Principal component analysis (PCA) was used to reduce the number of variables in the RR CARS hyperspectral dataset to a limited number of PC spectra that contain the majority of the variance in the data. The calculated PCs were ranked based on their total loading, or eigenvalue (EV). The number of relevant PCs was determined by selecting those PCs that accounted for $80 \%$ of the variance. A side benefit of PCA is reduction in spatially uncorrelated noise from the RR CARS dataset.

After PCA, a hierarchical cluster analysis (HCA) algorithm was used to identify Raman frequencies that showed strongly correlated loadings across multiple PCs. This approach was used to recognize intraspectral correlations, e.g., from Raman features coming from the same molecular class, rather than interspectral correlations among spectra obtained from different spatial locations, which is typically done with HCA (van Manen et al. 2005; Matthaus et al. 2007). Using PCs ensured the most robust identification of common peaks since substantial noise reduction occurred as a result of the PCA.

Clustering with the unweighted pair group method with arithmetic mean (UPGMA) algorithm was performed on the correlation matrix of the PC spectra using 1 minus the correlation as distance metric. Those frequencies with smallest distance were grouped, and the process was bootstrapped to bin spectral features correlated across the PC spectra, resulting in a dendrogram of Raman frequencies. Final clusters were defined by thresholding the dendrogram to retain clusters with distances smaller than a threshold distance, which was chosen empirically. Groups in the dendrogram with larger distances than the threshold were not included into any clusters. Finally, a single-cluster spectrum for each dataset was constructed by (1) computing the summed, normalized spectrum for the entire hyperspectral dataset and (2) scaling this spectrum at each frequency with the so-called communality value that accounts for the relative variance for a particular frequency element that was captured by the PCs used for clustering (see Eqs. 1 and 2, below).

$\mathrm{NS}(\omega)=\sum_{k=0}^{m} D_{k}(\omega)$

$\mathrm{CS}(\omega)=\sqrt{\sum_{j=1}^{n} \mathrm{PC}_{j}(\omega)^{2}} * \mathrm{NS}(\omega)$

where $\mathrm{NS}(\omega)$ is the normalized, summed spectrum at frequency $\omega$, computed by adding the values from each individual $m$ spectra (or equivalently, spatial positions) in the normalized RR CARS hyperspectral dataset $D$. The cluster spectrum, $\operatorname{CS}(\omega)$, is calculated by multiplying NS by the root mean square value at the corresponding $\omega$, summing over $n$ PC spectra used for clustering. This weighting factor scaled NS by the contribution (via harmonically summed loadings) of each frequency to the PC subset used for HCA clustering. Finally, HCA score for a single cluster at each spatial position was produced by projection of the normalized data $(D)$ on the cluster spectrum (see Eq. 3)

Score $=\sum_{i=1}^{q} D\left(\omega_{i}\right) * \operatorname{CS}\left(\omega_{i}\right)$

where $q$ is the number of Raman frequencies in the selected cluster, $D$ is the normalized RR CARS dataset, and CS is the value of cluster spectrum, respectively, at Raman frequency $\omega i_{\mathrm{i}}$.

Plotting scores at each $x-y$ location essentially forms a denoised, chemically specific image (called an HCA scores map) similar to that from peak integration of RR CARS data. The PCA and HCA in this work were done using ChemomeTricks toolbox for MATLAB version 7.0 (The MathWorks, Natick, MA) developed at FOM Institute AMOLF and will be described in detail in an upcoming publication.

\section{Results and discussion}

Tissue morphology shown by bright field, CARS, and fluorescence microscopy

The experimental procedure used to investigate the muscle tissues is outlined in the chart in Fig. 2a. As mentioned before, we use a model experimental system genetically modified to overexpress PLIN5 to stimulate LD formation upon receiving a high-fat diet. After killing rats, serial transverse sections in the following order were excised from the tibialis muscle: $20,5,5,5$, and $5 \mu \mathrm{m}$. The second $5-\mu \mathrm{m}$ section was stained to visualize lipids, cell borders (sarcolemma), and nuclei by immunofluorescence. This section was used as a histological scout image for the CARS measurements. All hyperspectral CARS microscopy data presented in this study were obtained from the first $5-\mu \mathrm{m}$ or $20-\mu \mathrm{m}$ section without further preparation. The resulting hyperspectral dataset was analyzed by first converting the raw CARS data into quantitative RR CARS data, after which two separate analyses were performed. Initially, chemically specific vibrational maps (images) were obtained from the RR CARS spectra by integration of specific Raman peaks. This yielded an image corresponding to the distribution of specific compounds. Next, HCA was performed and maps were plotted.

Figure $2 \mathrm{~b}$ shows the bright-field image of a $20-\mu \mathrm{m}-$ thick muscle section. As in typical transverse skeletal 
(A)

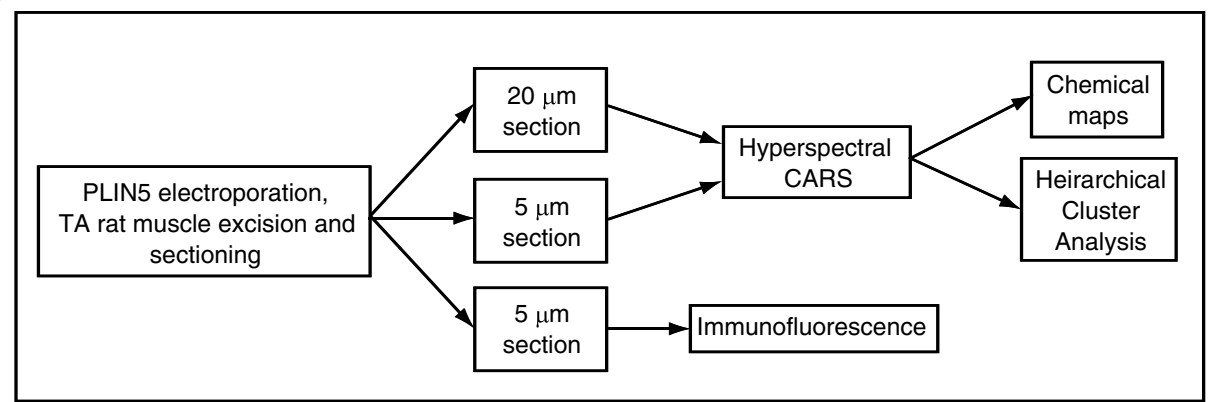

(B)

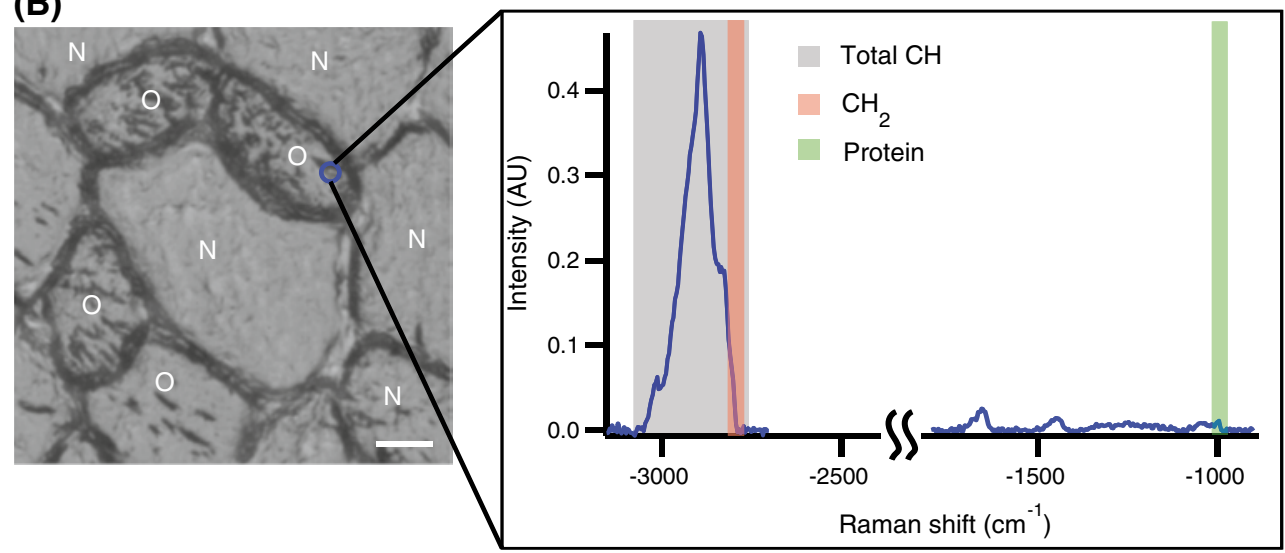

Fig. 2 a Schematic outline of the experimental procedure. b Brightfield image of a $20-\mu \mathrm{m}$-thick section of muscle tissue. $N$ and $O$ markings depict native and overexpressing fibers, respectively. Graph

muscle sections, the fibers appear as enclosed shapes with dark sarcolemmal borders. Visual inspection of the image indicates that fibers can be divided into two groups. Fibers from the first group, e.g., the large fiber in the center of the image, show generally uniform intensity within the fibers compared to the second group, which contains a large number of elongated dark features. Immunostaining of the third 5- $\mu \mathrm{m}$ slice for PLIN5 (not shown) revealed that the second group corresponds to fibers where PLIN5 was efficiently overexpressed. Hence, in the following text, the two groups of fibers will be referred to as "native" (labeled "N") or "overexpressing" (labeled "O") cells, respectively, for cells without and with PLIN5 overexpression. We note that native fibers express PLIN5 as well, albeit at a basal level that is much lower than upon overexpression (Bosma et al. 2012). Thus, we were able to take full advantage of the CARS imaging approach allowing detailed examination of LDs present in muscle fibers with native and abundant overexpression of PLIN5 within the same tissue sample. As such, native fibers effectively served as an internal control for comparison with overexpressing fibers.

As mentioned in the methods, a CARS spectrum from $-3,100$ to $-600 \mathrm{~cm}^{-1}$ is acquired at each spatial position in the image. After processing raw CARS data into RR shows an RR CARS spectrum from a single location in the image with highlighted spectral regions that are used in this study. Scale bar $20 \mu \mathrm{m}$

CARS spectra (see Methods section), whose signal intensity is proportional to molecular concentration (Fig. 2b, inset), chemical maps were generated to highlight cellular structures. Figure $3 \mathrm{a}$ is a map showing the concentration of all $\mathrm{CH}$ resonances in the tissue section consisting of $8 \times 8$ "tiles," as described in the Methods section. Since most organic material in tissue contains carbon and hydrogen, $\mathrm{CH}$ maps are used to provide an overview of cellular structures. High-intensity (white) deposits of $\mathrm{CH}$-rich structures were primarily observed in overexpressing fibers, and these deposits presumably contain lipid species since lipids contain the highest concentration of $\mathrm{CH}$ groups. Comparing the $\mathrm{CH}$ map with the immunofluorescence image from the histological scout shows that LDs (green) also appear predominantly in the same overexpressing cells (Fig. 3b). The coincidence of elevated $\mathrm{CH}$ signals in hyperspectral $\mathrm{RR}$ CARS data and the strong LD fluorescence confirms that overexpressing muscle fibers show abundant intracellular $\mathrm{CH}$ deposits, whereas the native fibers display uniform, rather low $\mathrm{CH}$ intensity. Interestingly, the low-contrast, elongated features within overexpressing fibers in bright field (Fig. 2b) also show relatively little $\mathrm{CH}$ contrast in the hyperspectral CARS map (Fig. 3a). This observation is discussed further below. 


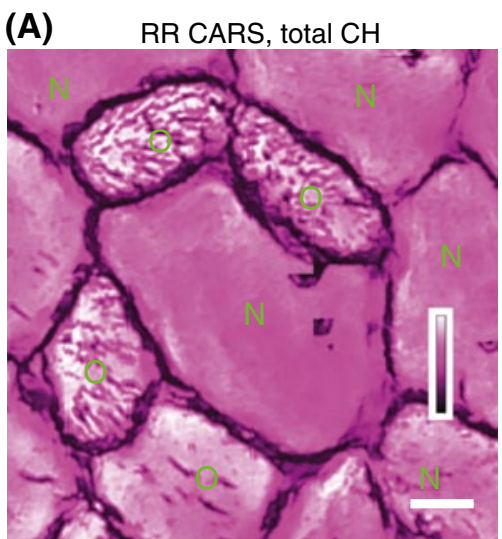

(B)

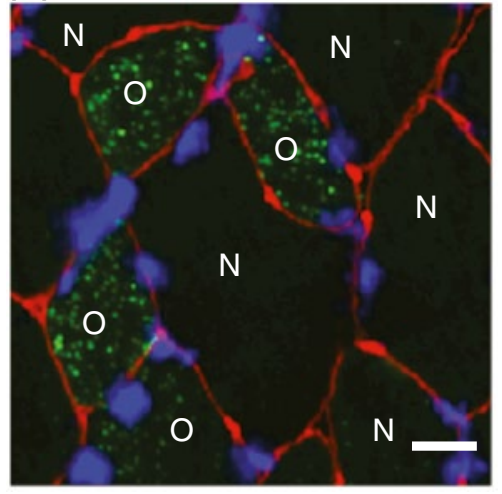

Fig. 3 a Intensity map of the integrated RR CARS spectra over the entire $\mathrm{CH}$ region $\left(-2,800\right.$ to $\left.-3,100 \mathrm{~cm}^{-1}\right)$ of the $20-\mu \mathrm{m}$ section; color bar shows the linear intensity scale b Immunofluorescence image of the corresponding region from the 5- $\mu \mathrm{m}$ "scout" section showing lipids (green), nuclei (blue), and sarcolemma (red). As in Fig. 2, $N$ and $O$ markings depict native and overexpressing fibers, respectively. Scale bar $=20 \mu \mathrm{m}$ in all images

HCA identification of LDs and chemical heterogeneity from CH CARS

Figure 3 identifies $\mathrm{CH}$-rich (and poor) structures in muscle fibers from inspection of a single chemical map binning all CH-related intensities $\left(-3,100\right.$ to $\left.-2,800 \mathrm{~cm}^{-1}\right)$ into pixel intensity values. Because the $\mathrm{CH}$ region contains a collection of overlapping vibrations arising from the $\mathrm{CH}, \mathrm{CH}$, and $\mathrm{CH} 3$ groups abundant in most biological compounds, we chose to focus on the $\mathrm{CH} 2$ vibration to highlight lipid molecules that typically have long acyl chains with many $\mathrm{CH} 2$ groups. A chemical map from the hyperspectral RR CARS data of this vibration $\left(-2,845 \mathrm{~cm}^{-1}\right)$ is presented in Fig. 4a. Note these data are from the corresponding $5-\mu \mathrm{m}$ section covering the same area as shown in Figs. 2 and 3 . The small punctate features with high intensity in Fig. $4 \mathrm{a}$ are LDs and have a maximum lateral diameter of 2-3 $\mu \mathrm{m}$, consistent with the fluorescence imaging in the scout sample. LDs in the PLIN5-overexpressing fibers are homogeneously distributed but are generally located in the vicinity of rod-like, low-intensity $\mathrm{CH} 2$ regions. Native fibers are characterized by a weaker and uniform $\mathrm{CH} 2$ response.

As an initial test for our chemometric analysis (see "Methods" section), we generated an HCA scores map for the strongly correlated cluster of frequencies between $-2,844$ and $-2,863 \mathrm{~cm}^{-1}$, essentially covering the $\mathrm{CH} 2$ vibrational mode. The HCA scores map (Fig. 4b) shows similar features with the $-2,845 \mathrm{~cm}^{-1}$ intensity map from the hyperspectral RR CARS data as well as other smaller, punctate lipid accumulations. LDs and sarcolemmal regions are visible in the HCA scores map with even higher contrast than compared to Fig. 4a, likely because of the noise reduction in the HCA process. Furthermore, the membrane-rich subsarcolemmal regions (Spangenburg et al. 2011) also show much stronger contrast as compared to the crude $\mathrm{CH} 2$ image plot.

When inspecting spectra from four different regions in the tissue (Fig. 4c), distinct, conserved features are seen in the spectra of LDs such as the $\mathrm{CH} 2$ shoulder at $-2,845 \mathrm{~cm}^{-1}$ and the peak at $-2,880 \mathrm{~cm}^{-1}$. Indeed, HCA scores maps from two other frequency clusters, $-2,871$ to $-2,876 \mathrm{~cm}^{-1}$ and $-2,880$ to $2,905 \mathrm{~cm}^{-1}$, show similar image features as Fig. 4b. When considering the molecular entities corresponding to these vibrational modes ( $\mathrm{CH} 2$ asymmetric $\left(2,874 \mathrm{~cm}^{-1}\right)$ and $\mathrm{CH} 2$ Fermi resonance $\left.\left(2,888 \mathrm{~cm}^{-1}\right)\right)$ and the biochemistry of long acyl chains in lipids, this observation makes sense. RR CARS spectra from LDs in cultured adipocytes also show both of these features (Rinia et al. 2008). Coincidence of HCA image features (Fig. 4b) with those in the chemical map obtained by direct integration of the $\mathrm{CH} 2$ mode in the RR CARS data (Fig. 4a) strongly supports the applicability of chemometric analysis to hyperspectral RR CARS data for chemical imaging of as-prepared tissue sections.

HCA scores maps reveal mitochondria-like features in overexpressing fibers

Up to this point, our analysis has focused solely on the $\mathrm{CH}$ region $\left(-3,100\right.$ to $\left.-2,800 \mathrm{~cm}^{-1}\right)$ of the hyperspectral $R R$ CARS data. However, RR CARS spectra contain numerous other resonances, specifically in the fingerprint region of the vibrational spectrum $\left(-700\right.$ to $\left.-1,750 \mathrm{~cm}^{-1}\right)$, which have been assigned to specific macromolecular classes, e.g., proteins, nucleic acids, and lipids. Not unlike the $\mathrm{CH}$ region, these peaks often overlap, so HCA analysis should be useful tool to decipher recurring patterns in the sample. In Fig. 5, we show images from HCA scores maps and chemical maps from modes in the fingerprint region to highlight cellular structures with distinct biochemistry.

Using hyperspectral RR CARS data from the $20-\mu \mathrm{m}$ section, HCA systematically returned a set of three 
(A)

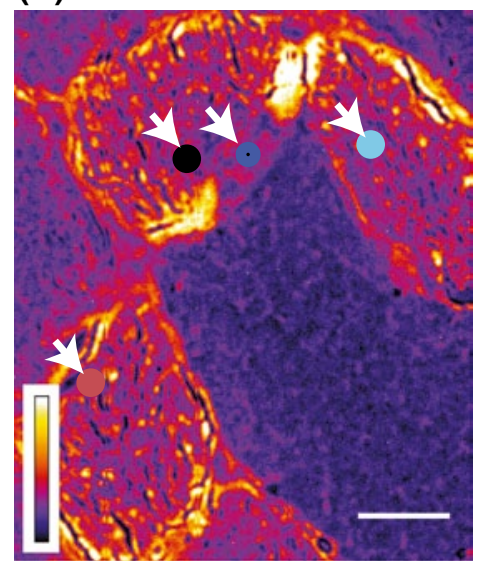

(B)

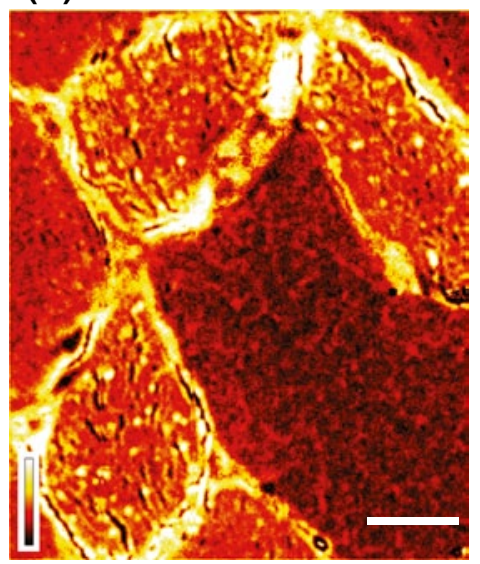

(C)

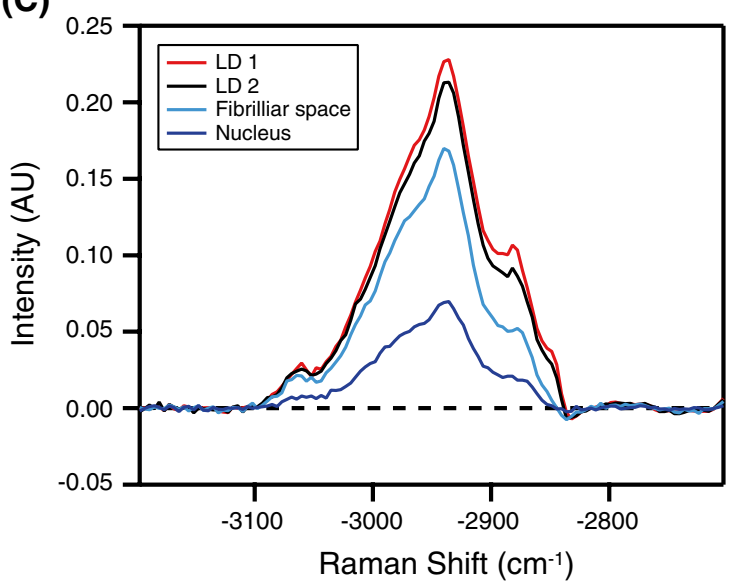

Fig. 4 a Intensity map of the integrated RR CARS spectra over the $-2,845 \mathrm{~cm}^{-1}$ peak (symmetric $\mathrm{CH} 2$ stretch) in a $5-\mu \mathrm{m}$-thick tissue section. b HCA scores map of the cluster of frequencies between $-2,844$ and $-2,863 \mathrm{~cm}^{-1}$ for the same tissue as in a. c RR CARS spectra in the $\mathrm{CH}$ region at positions within the sample corresponding to LDs (red and black), myofibrillar space (light blue), and nucleus (dark blue). The locations where the spectra were collected are highlighted and indicated with arrows in a. Each spectrum is averaged over five neighboring pixels, covering an area of approximately $1 \times 1 \mu \mathrm{m}^{2}$. Scale bar $20 \mu \mathrm{m}$ in all images; color bars show the linear intensity scales in panels $\mathbf{a}$ and $\mathbf{b}$

is expected from within the muscle fibers as reflected in Fig. 5b; however, the elongated features are only visible with positive contrast after multivariate analysis. This is likely due to refractive index mismatches and scattering that reduces the overall CARS intensity from these locations (supported by their low contrast in bright field in Fig. 2b). This demonstrates the ability of chemometric analysis applied to hyperspectral RR CARS data to decipher subtle changes in otherwise noisy or highly inhomogeneous datasets.

Figure 5d shows the HCA scores map of the $\mathrm{CH} 2$ symmetric cluster (from $-2,844$ to $-2,863 \mathrm{~cm}^{-1}$ ) for this tissue section, which again specifically highlights LDs and lipid-rich regions. The LDs appear mostly in overexpressing fibers, similar to the protein-rich structures. An overlay of the HCA image of proteins (Fig. 5a) and the LD image (Fig. 5d) shows that the protein-rich structures colocalize/ border the LDs (Fig. 5e). Based on our immunofluorescence staining of mitochondria and LDs in PLIN5-overexpressing tissues (Supplemental Material), the morphological appearance of the elongated protein-rich structures, and the evidence that protein concentration in the mitochondrial matrix is 1.5-fold higher than the cytoplasm (Srere 1980; Soboll et al. 1976), we suggest that the protein-rich features near LDs represent mitochondria.

Unlike immunofluorescence imaging, no exogenous labels were used in Fig. 5 to identify LDs or protein-rich features as this was accomplished solely based on inherent chemical compounds present in these organelles as measured by hyperspectral CARS imaging. While additional experiments, such as a correlative study of fluorescence and hyperspectral CARS, are required to definitively identify 

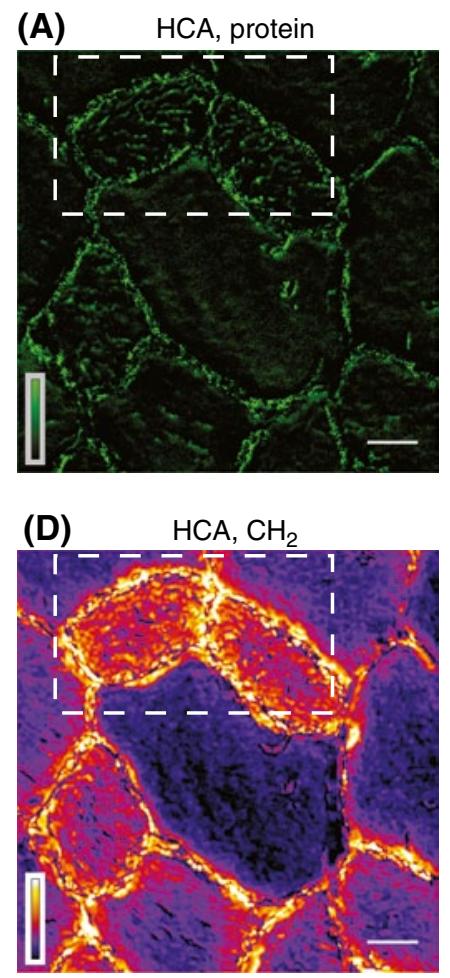

(B) RR CARS, protein

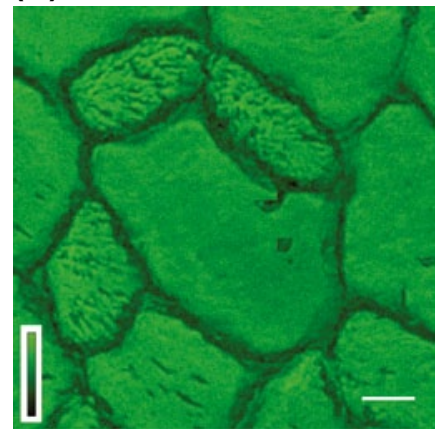

(E)
(C)

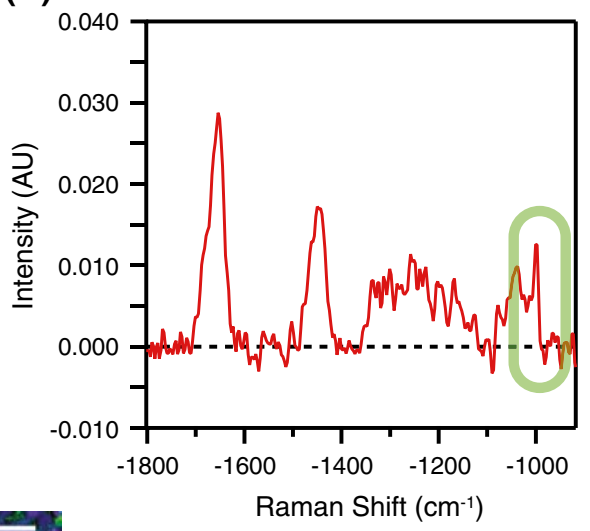

Raman Shift $\left(\mathrm{cm}^{-1}\right)$

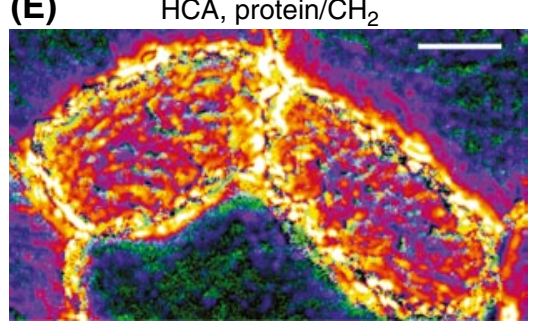

Fig. 5 a HCA scores map of the cluster of frequencies between $-1,000$ and $-1,003 \mathrm{~cm}^{-1}$ of the 20 - $\mu \mathrm{m}$-thick tissue section. b Intensity map of the integrated RR CARS spectra over the $-1,002 \mathrm{~cm}^{-1}$ peak, presumably showing the distribution of phenylalanine. c Average fingerprint RR CARS spectrum over all image pixels in $\mathbf{b}$ reveals

the protein-rich features as mitochondria, the results presented here combined with our previous work (Bosma et al. 2012) constitute strong evidence that HCA of the protein band revealed features representing mitochondria in the vicinity of the LDs.

\section{Conclusions}

Hyperspectral CARS microscopy was shown to be a labelfree and non-invasive method to simultaneously localize macromolecules and cellular organelles in biochemically complex tissue samples without complex sample preparation. Using chemical imaging of specific vibrational modes, we showed lipid accumulations in PLIN5-overexpressing muscle fibers of freshly prepared tissue sections. Additional chemometric analysis with HCA enhanced image contrast for lipid accumulations and revealed mitochondrialike features in overexpressing fibers that were otherwise unobservable with direct peak integration of hyperspectral RR CARS data. Taken together, these results demonstrate identification of organelle interaction with LDs in biochemically heterogeneous tissue environments using purely vibrational contrast. a clear peak at $-1,002 \mathrm{~cm}^{-1}$ (marked region). d HCA scores map of the cluster of frequencies between $-2,844$ and $-2,863 \mathrm{~cm}^{-1}$ highlighting LDs. e Zoomed overlay of $\mathbf{a}$ and $\mathbf{d}$ for the area indicated showing colocalization of protein structures (green) with the borders of the LDs (bright yellow). Scale bar $20 \mu \mathrm{m}$ in all images

While the resolving power of hyperspectral CARS is limited compared to electron microscopy and the macromolecular identification suffers compared to mass spectrometry, the data presented here demonstrate that hyperspectral CARS data can be processed to highlight features similar to those seen in electron micrographs without extensive supervision or sample preparation (e.g., immunogold labeling, critical-point dehydration, matrix deposition). Furthermore, CARS imaging did not suffer from the same challenges as Raman microscopy, and we were able to successfully obtain data of sufficient quality for reliable organelle identification with both $\mathrm{CH}$ and fingerprint spectra.

Future studies will focus on using hyperspectral CARS for the determination of the chemical composition of individual LDs within muscle tissues to identify whether overexpressing fibers contain different neutral lipid species than those in native tissues. The use of CARS microscopy provides a complementary capability to localize biological components within tissues based on intrinsic sample biochemistry without laborious sample preparation and should be useful as diagnostic platform in tissue pathogenesis.

Acknowledgments This study was financially supported by the Nederlandse Organisatie voor Wetenschappelijk Onderzoek 
(Netherlands Organization for the Advancement of Research) (M.B., G.R.), the NanoNextNL, a micro and nanotechnology consortium of the Government of the Netherlands and 130 partners (N.B., M.B., and M.K.C.L.), and a Marie Curie Foundation grant \#CIG322284 (S.H.P). Ma.B. was financially supported by NUTRIM and the Graduate School VLAG. A Vici (Grant 918.96.618) grant for innovative research from the Netherlands Organization for Scientific Research supports the work of P.S.

G.R. and N.B. performed CARS experiments. P.L. and G.H. helped construct the experimental system. GR and GE did the multivariate analysis using HCA and PCA. A.G., Ma.B., and N.B. performed the fluorescence imaging. N.B., Ma.B., M.K.C.H., and P.S. provided samples. G.R., N.B., M.B., and S.H.P. wrote the paper. M.B. and S.H.P. supervised the research. All authors contributed to discussion of the results and revision of the paper. The authors wish to thank Dr. E. Cánovas and Dr. W. Rock for stimulating discussions and technical support.

\section{References}

Aguer C, Mercier J, Man CYW, Metz L, Bordenave S, Lambert K, Jean E, Lantier L, Bounoua L, Brun JF, de Mauverger ER, Andreelli F, Foretz M, Kitzmann M (2010) Intramyocellular lipid accumulation is associated with permanent relocation ex vivo and in vitro of fatty acid translocase (FAT)/CD36 in obese patients. Diabetologia 53(6):1151-1163. doi:10.1007/s00125-010-1708-x

Bosma M, Minnaard R, Sparks LM, Schaart G, Losen M, de Baets MH, Duimel H, Kersten S, Bickel PE, Schrauwen P, Hesselink MKC (2012) The lipid droplet coat protein perilipin 5 also localizes to muscle mitochondria. Histochem Cell Biol 137(2):205216. doi:10.1007/s00418-011-0888-x

Boxer SG, Kraft ML, Weber PK (2009) Advances in imaging secondary ion mass spectrometry for biological samples. Annu Rev Biophys 38(1):53-74. doi:10.1146/annurev.biophys.050708.133634

Centonze VE, White JG (1998) Multiphoton excitation provides optical sections from deeper within scattering specimens than confocal imaging. Biophys J 75(4):2015-2024

Chavez JA, Knotts TA, Wang LP, Li G, Dobrowsky RT, Florant GL, Summers SA (2003) A role for ceramide, but not diacylglycerol, in the antagonism of insulin signal transduction by saturated fatty acids. J Biol Chem 278(12):10297-10303. doi:10.1074/jbc. M212307200

Day JPR, Rago G, Domke KF, Velikov KP, Bonn M (2010) Label-free imaging of lipophilic bioactive molecules during lipid digestion by multiplex coherent anti-Stokes Raman scattering microspectroscopy. J Am Chem Soc 132(24):8433-8439. doi:10.1021/ja102069d

Fletcher JS, Vickerman JC (2013) Secondary ion mass spectrometry: characterizing complex samples in two and three dimensions. Anal Chem 85(2):610-639. doi:10.1021/Ac303088m

Gawlik KI, Durbeej M (2011) Skeletal muscle laminin and MDC1A: pathogenesis and treatment strategies. Skelet Muscle 1(1):9. doi:10.1186/2044-5040-1-9

Goodpaster BH, Theriault R, Watkins SC, Kelley DE (2000) Intramuscular lipid content is increased in obesity and decreased by weight loss. Metab Clin Exp 49(4):467-472. doi:10.1016/ s0026-0495(00)80010-4

Goodpaster BH, He J, Watkins S, Kelley DE (2001) Skeletal muscle lipid content and insulin resistance: evidence for a paradox in endurance-trained athletes. J Clin Endocrinolo Metab 86(12):5755-5761

Helmchen F, Denk W (2005) Deep tissue two-photon microscopy. Nat Methods 2(12):932-940. doi:10.1038/nmeth818

Lee D-E, Kehlenbrink S, Lee H, Hawkins M, Yudkin JS (2009) Getting the message across: mechanisms of physiological cross talk by adipose tissue. Am J Physiol Endocrinol Metab 296(6):E1210-E1229. doi:10.1152/ajpendo.00015.2009

Liu Y, Lee YJ, Cicerone MT (2009) Broadband CARS spectral phase retrieval using a time-domain Kramers-Kronig transform. Opt Lett 34(9):1363-1365

Matthaus C, Chernenko T, Newmark JA, Warner CM, Diem M (2007) Label-free detection of mitochondrial distribution in cells by nonresonant Raman microspectroscopy. Biophys J 93(2):668-673. doi:10.1529/biophysj.106.102061

Matthaus C, Krafft C, Dietzek B, Brehm BR, Lorkowski S, Popp J (2012) Noninvasive imaging of intracellular lipid metabolism in macrophages by Raman Microscopy in combination with stable isotopic labeling. Anal Chem 84(20):8549-8556. doi: $10.1021 / \mathrm{ac} 3012347$

Nielsen J, Mogensen M, Vind BF, Sahlin K, Hojlund K, Schroder HD, Ortenblad N (2010) Increased subsarcolemmal lipids in type 2 diabetes: effect of training on localization of lipids, mitochondria, and glycogen in sedentary human skeletal muscle. Am J Physiol Endocrinol Metab 298(3):E706-E713. doi:10.1152/ajpe ndo.00692.2009

Pohling C, Buckup T, Motzkus M (2011) Hyperspectral data processing for chemoselective multiplex coherent anti-Stokes Raman scattering microscopy of unknown samples. J Biomed Opt 16(2). doi:10.1117/1.3533309

Pohling C, Buckup T, Pagenstecher A, Motzkus M (2011b) Chemoselective imaging of mouse brain tissue via multiplex CARS microscopy. Biomed Optics Express 2(8):2110-2116. doi:10.13 64/BOE.2.002110

Puppels GJ, Demul FFM, Otto C, Greve J, Robertnicoud M, Arndtjovin DJ, Jovin TM (1990) Studying single living cells and chromosomes by confocal Raman microspectroscopy. Nature 347(6290):301-303. doi:10.1038/347301a0

Rinia HA, Burger KNJ, Bonn M, Muller M (2008) Quantitative labelfree imaging of lipid composition and packing of individual cellular lipid droplets using multiplex CARS microscopy. Biophys J 95(10):4908-4914. doi:10.1529/biophysj.108.137737

Rompp A, Spengler B (2013) Mass spectrometry imaging with high resolution in mass and space. Histochem Cell Biol 139(6):759783. doi:10.1007/s00418-013-1097-6

Seppanen-Laakso T, Laakso I, Hiltunen R (2002) Analysis of fatty acids by gas chromatography, and its relevance to research on health and nutrition. Anal Chim Acta 465(1-2):39-62. doi:10.1016/s0003-2670(02)00397-5

Shaw CS, Jones DA, Wagenmakers AJ (2008) Network distribution of mitochondria and lipid droplets in human muscle fibres. Histochem Cell Biol 129(1):65-72. doi:10.1007/s00418-007-0349-8

Soboll S SR, Freisl M, Elbers R, Heldt HW (1976). In: JM Tager HS, JR Williamson (ed) Use of Isolated Liver Cells and Kidney Tubules in Metabolic Studies, North-Holland, Amsterdam and Oxford, pp 29-40

Sollner TH (2007) Lipid droplets highjack SNAREs. Nat Cell Biol 9(11):1219-1220. doi:10.1038/ncb1107-1219

Spangenburg EE, Pratt SJP, Wohlers LM, Lovering RM (2011) Use of BODIPY (493/503) to Visualize Intramuscular Lipid Droplets in Skeletal Muscle. J Biomed Biotechnol. doi:10.1155/2011/598358

Srere PA (1980) The infrastructure of the mitochondrial matrix. Trends in biochemical sciences 5(5):120-121. doi:http://dx.doi. org/10.1016/0968-0004(80)90051-1

Stratford S, Hoehn KL, Liu F, Summers SA (2004) Regulation of insulin action by ceramide: dual mechanisms linking ceramide accumulation to the inhibition of Akt/protein kinase B. J Biol Chem 279(35):36608-36615. doi:10.1074/jbc.M406499200

Tolles WM, Nibler JW, McDonald JR, Harvey AB (1977) Review of theory and application of coherent anti-stokes Ramanspectroscopy (CARS). Appl Spectrosc 31(4):253-271. doi:10.1366/0003702777744663625 
Vaandrager AB, Testerink N, Ajat M, Houweling M, Brouwers J, Pully VV, van Manen HWJ, Otto C, Helms JB (2009) Raman imaging and lipidomic analysis of lipid droplets in (activated) hepatic stellate cells. Chem Phys Lipids 160:S7-S8. doi:10.1016/j.chemphyslip.2009.06.109

van Manen HJ, Kraan YM, Roos D, Otto C (2005) Single-cell Raman and fluorescence microscopy reveal the association of lipid bodies with phagosomes in leukocytes. Proc Natl Acad Sci USA 102(29):10159-10164. doi:10.1073/pnas.0502746102

Vartiainen EM, Rinia HA, Muller M, Bonn M (2006) Direct extraction of Raman line-shapes from congested CARS spectra. Opt Express 14(8):3622-3630. doi:10.1364/oe.14.003622
Wang H, Zhao J, Lee AM, Lui H, Zeng H (2012) Improving skin Raman spectral quality by fluorescence photobleaching. Photodiagn Photodyn Ther 9(4):299-302. doi:10.1016/j.pdpdt.2012.02.001

Weigert R, Sramkova M, Parente L, Amornphimoltham P, Masedunskas A (2010) Intravital microscopy: a novel tool to study cell biology in living animals. Histochem Cell Biol 133(5):481-491. doi:10.1007/s00418-010-0692-z 